\title{
Leneadrement des recherches sur les cellules souches humaines \\ au crible de lonalyse parlementaire
}

Bénédicte de Boischevalier

L'Office parlementaire d'évaluation des choix scientifiques et technologiques (OPECST) a adopté à l'unanimité un rapport rédigé par le député Alain Claeys portant sur les recherches menées en France et en Europe sur les cellules souches embryonnaires humaines [1]. Ce rapport avait été demandé à I'OPECST par l'Assemblée Nationale en 2004, dans le cadre de la préparation des débats du Parlement qui «devra en effet, au plus tard en 2009 comme cela est prévu dans la loi de bioéthique de 2004, se saisir à nouveau de la question de la recherche sur l'embryon et décider si la transposition nucléaire peut être autorisée en France » [1].

\section{Un certain nombre de recommandations}

Après de nombreuses auditions et visites à travers le monde, le député fait un certain nombre de recommandations.

- La loi de bioéthique de 2004 interdit les recherches sur l'embryon, mais les autorise, à titre dérogatoire, lorsqu'elles «sont susceptibles de permettre des progrès thérapeutiques majeurs... ». Inutile pour le député d'attendre 2009 et la nouvelle révision des lois de bioéthique de 2004 pour autoriser la recherche sur l'embryon strictement contrôlée par l'Agence de biomédecine. De même la transposition nucléaire, terme qu'Alain Claeys a préféré dans ce rapport à celui de «clonage thérapeutique » qu'il juge fallacieux, doit être permise dans les mêmes conditions. L'origine des ovocytes et le risque de marchandisation du corps de la femme restant des problèmes majeurs et entiers, un débat devra être organisé par l'Agence de Biomédecine portant sur le don d'ovocytes pour la recherche. Le rapport propose une action internationale en faveur de la réglementation du

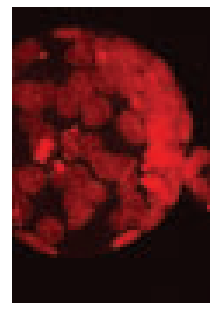

DRCT, Mission Éthique, Inserm, 101 , rue de Tolbiac, 75654 Paris Cedex 13, France. benedicte.de-boischevalier@ tolbiac.inserm.fr

don d'ovocytes pour la recherche afin de lutter contre l'exploitation des femmes. La provenance des ovocytes devrait être obligatoirement indiquée dans les publications internationales. De plus, le débat public devra permettre de valider certains principes: interdiction du don par des mineures, conditions du consentement éclairé, gratuité du don, remboursement des frais occasionnés par ce don et compensation des salaires non perçus, recueil des ovocytes uniquement dans des centres publics, suivi médical du don remboursé à $100 \%$ (ce recueil nécessite, en l'état actuel des recherches, de pratiquer une hyperstimulation ovarienne, qui n'est pas sans risques pour les femmes), séparation des centres de recueil et des laboratoires de recherche. Dans ce cadre, la ratification de la convention d'Oviedo [2, 3] de 1997 du Conseil de l'Europe qui stipule que «lorsque la recherche sur les embryons in vitro est admise par la loi, celle-ci assure une protection adéquate de l'embryon » doit être prudemment réexaminée en particulier au regard de sa compatibilité avec la transposition nucléaire.

- Aucun brevet ne devrait pouvoir être pris tant sur les cellules souches adultes que sur les cellules souches embryonnaires, seuls les procédés et les produits d'application pourront être brevetables (voir à ce sujet l'avis 93 du CCNE [4]).

- Le rapport suggère également de nouvelles mesures d'encadrement de la recherche sur les cellules souches 
embryonnaires. Afin de lutter contre la fraude scientifique comme celle de 2005 [5], il serait nécessaire de supprimer à vie l'obtention de fonds publics pour tout chercheur convaincu de manquement à la déontologie scientifique. Une formation à l'éthique de la recherche devrait faire obligatoirement partie du cursus de tous les étudiants.

- Le rapport ne néglige pas la dimension d'application en biotechnologie des découvertes potentielles des travaux menés sur les cellules souches. II recommande d'organiser une réflexion sur le statut du vivant en particulier en ce qui concerne la brevetabilité [4]. Pour assurer l'accessibilité à une ressource précieuse, le rapport suggère de créer dès maintenant des biobanques publiques de sang du cordon ombilical afin de limiter le développement des banques privées qui s'opposent au principe de solidarité.

- Le rapport regrette l'absence de données permettant de recenser les recherches françaises sur tous les types de cellules souches, d'origine humaine ou animale et de tous les financements privés et publics de ces recherches. II demande en conséquence à l'Agence d'établir un rapport annuel public destiné au parlement évaluant l'avancement des recherches en France ou à l'étranger sur les cellules souches.

- Le financement de la recherche est évidemment un point essentiel. L'Agence Nationale de Recherche doit afficher ses priorités dans ce domaine et une réflexion sur la création de pôles d'excellence doit être menée. Quelques domaines spécifiques de recherche devraient, d'après le Rapport, être mieux explorés afin d'éclairer les futures décisions des politiques et éventuellement résoudre certains des problèmes éthiques actuels: recherches sur la possibilité de la dérivation d'ovocytes à partir de cellules souches embryonnaires, une étude épidémiologique sur les conséquences de l'hyperstimulation ovarienne et enfin des études sur les conséquences sociales de la thérapie cellulaire.

\section{Pour conclure}

Dans sa conclusion, Alain Claeys revient sur le fait que la recherche sur les cellules souches comporte encore bien des incertitudes et des ignorances qui ne peuvent être comblées que par un gros effort de recherche fondamentale, recherche qui nécessitera des financements importants autant pour les cellules souches embryonnaires que pour les cellules souches adultes. $\diamond$

The framing on human stem cells research under the screen of French Parliament

\section{RÉFÉRENCES}

1. Office Parlementaire d'évaluation des choix scientifiques et technologiques, Rapport sur le fonctionnement des cellules vivantes par Alain Claeys, Député, décembre 2006.

http://www.assemblee-nationale.fr/12/pdf/rap-off/i3498.pdf

2. Conseil de l'Europe, Comité Directeur pour la Bioéthique, Convention sur les droits de l'homme et la biomédecine, 1997. http://conventions.coe.int/Treaty/fr/Treaties/Word/164.doc

3. Chneiweiss H. Sur les sentiers escarpés des montagnes de bio-éthique. Épisode 1 : les aiguilles du clonage. Med Sci (Paris) 2003 ; $19: 246-7$.

4. CCNE. Avis $n^{\circ} 93$. Commercialisation des cellules souches humaines et autres lignées cellulaires, 17 novembre 2006.

http://www.ccneethique.fr/francais/avis/a_093.htm5

5. Chneiweiss H. Cloningate? La publication scientifique et le clonage thérapeutique face à la mystification Hwang. Med Sci (Paris) 2006 ; 22 : 218-22.

\section{TIRÉS À PART}

B. de Boischevalier

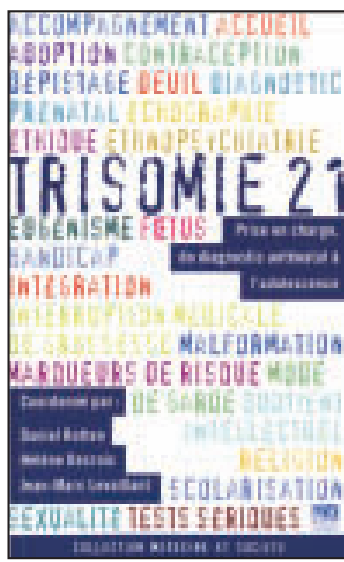

ISBN : 2-84254-105-7 248 pages

\section{Bon de commande}

À retourner à EDK, 2, rue Troyon - 92316 Sèvres Cedex Tél. : 0155641393 - Fax : 0155641394 - E-mail : edk@edk.fr

NOM : Prénom

Adresse

Code postal : Ville :

Pays :

Fonction :

Je souhaite recevoir l'ouvrage Trisomie 21: $15 €+3 €$ de port $=\mathbf{1 8} €$ TTC

en ................ exemplaire, soit un total de .................................... €

$\square$ Par chèque, à l'ordre de $\mathbf{E} \mathbf{D} \mathbf{K}$

Par carte bancaire : $\square$ Visa $\square$ Eurocard/Mastercard

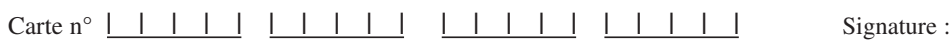

Date d'expiration: $\quad$ । $11 \quad$ । $1 \mid$

$\mathrm{N}^{\circ}$ de contrôle au dos de la carte : $\quad$ | । | | 